Review Article

\title{
Transcatheter versus Surgical Aortic Valve Replacement after Previous Cardiac Surgery: A Systematic Review and Meta-Analysis
}

\author{
Sharaf-Eldin Shehada $\mathbb{D}^{1},{ }^{1}$ Yacine Elhmidi $\left(\mathbb{D},{ }^{2}\right.$ Öznur Öztürk $\left(\mathbb{D},{ }^{3}\right.$ Markus Kasel ${ }^{D},{ }^{4}$ \\ Antonio H. Frangieh, ${ }^{4}$ Fanar Mourad (D), Jaroslav Benedik (D), ${ }^{1}$ Jaafar El Bahi, ${ }^{1}$ \\ Mohamed El Gabry, ${ }^{1}$ Matthias Thielmann, ${ }^{1}$ Heinz Jakob, ${ }^{1}$ and Daniel Wendt $\mathbb{D}^{1}$ \\ ${ }^{1}$ Department of Thoracic and Cardiovascular Surgery, West-German Heart and Vascular Center Essen, \\ University of Duisburg-Essen, Duisburg, Germany \\ ${ }^{2}$ Department of Cardiovascular Surgery, German Heart Center Munich, Technical University Munich, Munich, Germany \\ ${ }^{3}$ Department of Cardiovascular Surgery, Klinikum Bogenhausen, Munich, Germany \\ ${ }^{4}$ Department of Cardiology, German Heart Center Munich, Technical University Munich, Munich, Germany
}

Correspondence should be addressed to Sharaf-Eldin Shehada; sharaf.shehada@live.com

Sharaf-Eldin Shehada and Yacine Elhmidi equally contributed to this work.

Received 16 August 2017; Revised 27 December 2017; Accepted 23 January 2018; Published 5 April 2018

Academic Editor: Syed Wamique Yusuf

Copyright (c) 2018 Sharaf-Eldin Shehada et al. This is an open access article distributed under the Creative Commons Attribution License, which permits unrestricted use, distribution, and reproduction in any medium, provided the original work is properly cited.

\begin{abstract}
Aim. Aortic valve replacement (AVR) in patients with prior cardiac surgery might be challenging. Transcatheter aortic valve replacement (TAVR) offers a promising alternative in such patients. We therefore aimed at comparing the outcomes of patients with aortic valve diseases undergoing TAVR versus those undergoing surgical AVR (SAVR) after previous cardiac surgery. Methods and Results. MEDLINE, EMBASE, and the Cochrane Central Register were searched. Seven relevant studies were identified, published between $01 / 2011$ and $12 / 2015$, enrolling a total of 1148 patients with prior cardiac surgery (97.6\% prior CABG): $49.2 \%$ underwent TAVR, whereas $50.8 \%$ underwent SAVR. Incidence of stroke $(3.8$ versus $7.9 \%, p=0.04)$ and major bleeding ( 8.3 versus $15.3 \%, p=0.04$ ) was significantly lower in the TAVR group. Incidence of mild/severe paravalvular leakage $(14.4 / 10.9$ versus $0 \%, p<0.0001)$ and pacemaker implantation ( 11.3 versus $3.9 \%, p=0.01)$ was significantly higher in the TAVR group. There were no significant differences in the incidence of acute kidney injury (9.7 versus $8.7 \%, p=0.99$ ), major adverse cardiovascular events ( 8.7 versus $12.3 \%, p=0.21$ ), 30 -day mortality (5.1 versus $5.5 \%, p=0.7$ ), or 1 -year mortality (11.6 versus $11.8 \%, p=0.97$ ) between the TAVR and SAVR group. Conclusions. TAVR as a redo procedure offers a safe alternative for patients presenting with aortic valve diseases after previous cardiac surgery especially those with prior CABG.
\end{abstract}

\section{Introduction}

Since decades, surgical aortic valve replacement (SAVR) has been considered as the gold standard for patients presenting with severe aortic stenosis (AS) [1]. SAVR can be performed either through conventional or minimal access methods $[2,3]$. Patients with prior cardiac surgery and symptomatic aortic stenosis, especially those patients with previous coronary artery bypass grafting (CABG), were at higher risk. Transcatheter aortic valve replacement (TAVR) has been established as an equivalent alternative to surgical AVR in high-risk patients. Moreover, TAVR is currently evaluated even in intermediate-risk patients $[4,5]$. Especially in the redo situation, TAVR decreases the risk of patent graft injury, which has been reported to be as high as $5 \%[6,7]$. While the use of TAVR is increasing worldwide, there is a current debate whether TAVR is superior to conventional SAVR in patients with previous cardiac surgery. Only few studies have been published comparing either the results of TAVR only [8-13] or SAVR only [14-17] in patients 
presenting with prior cardiac surgery. Some other studies matched and compared the results of both treatment modalities in redo patients [18-24]. Therefore, the purpose of this meta-analysis was to compare the outcomes in patients with prior cardiac surgery who underwent TAVR versus a conventional SAVR.

\section{Methods}

2.1. Data Collection and Inclusion Criteria. Based on the PRISMA guidelines [25], MEDLINE, EMBASE, and the Cochrane Central Register of Controlled Trials were searched from December 2015. Two investigators (Sharaf-Eldin Shehada and Yacine Elhmidi) independently assessed the relevant publications for eligibility through the title or the abstract of each publication. Only studies and articles using the following medical subject heading terms were evaluated: transcatheter aortic valve implantation/replacement, surgical/conventional aortic valve replacement, aortic valve stenosis, prior/previous cardiac surgery, previous coronary artery bypass grafting, and aortic valve replacement as a redo procedure after cardiac surgery. References of all relevant articles were also included in an additional search.

Inclusion criteria were as follows: (1) articles published between January 2011 and December 2015, (2) studies evaluating the impact of previous cardiac surgery especially those with a history of coronary artery bypass grafting in patients with severe aortic stenosis, (3) articles which compared TAVR and SAVR after prior CABG, (4) only studies including at least 40 patients in each group, (5) studies presenting VARC criteria, and (6) only articles written in English language.

2.2. Definition of Outcomes. Outcomes are defined based on the included studies, whereas only evaluated endpoints in the initial studies were collected and evaluated for this metaanalysis. The primary endpoints of our meta-analysis were as follows: (1) early (defined as inhospital or 30-day mortality based on the included studies), one-year mortality, and overall mortality (defined as all-cause mortality at the time of follow-up in each individual study, which varies between 6 and 48 months), (2) incidence of stroke, (3) acute kidney injury, and (4) major adverse cardiovascular events (MACE), according to VARC II [26]. Secondary endpoints included (1) incidence of major bleeding (including operative revision), (2) incidence of pacemaker implantation, (3) incidence of paravalvular leakage, (4) procedural times, and (5) the length of hospital stay.

2.3. Statistical Analysis. Continuous variables were expressed as mean \pm standard deviation $(\mathrm{SD})$ or median with interquartile range (IQR) (25-75th percentiles). Categorical variables were presented as numbers and percentages. The meta-analysis was performed using the Review Manager 5.3 software package (Nordic Cochrane Centre, Copenhagen, Denmark). Pooled estimation of odds ratios (ORs) with their 95\% confidence intervals (CIs) was calculated using the Mantel-Haenszel method in cases of absence of heterogeneity between the compared studies. Heterogeneity of the studies was assessed with the $I^{2}$ index, which indicates 25\%,50\%, and $75 \%$ as low, moderate, and high heterogeneity, respectively. If significant heterogeneity between the studies was detected, the DerSimonian and Laird random-effect methods were used. Sensitivity analysis was performed by eliminating each study at a time to assess the influence of any included study on the results. All reported $P$-values are two-sided, and a value of $P<0.05$ was considered statistically significant.

\section{Results}

The primary search revealed 346 potential relevant studies and articles. After removal of nonrelevant articles, a total of 21 studies remained. Three studies were excluded, as they did not fulfill the inclusion criteria (published after December 2015). Hence, 18 studies were evaluated. After final exclusion, seven studies remained for the systematic meta-analysis (Figure 1). These seven studies compromised a total of 1148 patients with a history of previous cardiac surgery (coronary artery bypass grafting in 1121 (97.6\%) patients), of whom $565(49.2 \%)$ underwent TAVR and 583 (50.8\%) underwent SAVR. Table 1 summarizes the total incidences of the endpoints of the metaanalysis, and all baseline demographics and echocardiographic data of the included studies were summarized in Table 2. Patients enrolled were mainly males and nearly a half had diabetes. Overall, about $50 \%$ of patients presented with peripheral vascular disease. Patients' age ranged from $78.1 \pm 5$ to $82 \pm 5.8$ years in the TAVR cohort versus $70.6 \pm 8$ to $82.3 \pm$ 6.2 years in the SAVR cohort. The STS PROM and logistic EuroSCORE ranged from $7.3 \pm 2.7 \%$ to $24 \pm 6 \%$ and $11.1 \pm$ $2.8 \%$ to $36.4 \pm 17.4 \%$ in the TAVR cohort versus $6.3 \pm 6 \%$ to $19 \pm 6 \%$ and $10.4 \pm 3 \%$ to $33.8 \pm 15.3 \%$ in the SAVR cohort, respectively.

There was no difference in early mortality $(5.1 \%$ in TAVR versus 5.5\% in SAVR patients: OR 0.89 (95\% CI 0.49 to 1.62$\left.) ; p_{\text {heterogeneity }}=0.7 ; I^{2}=12 \%\right)$, without significant heterogeneity among the studies (Figure 2(a)); one-year mortality (11.6\% versus $11.8 \%$, OR 1.01 (95\% CI 0.59 to $\left.1.72) ; \quad p_{\text {heterogeneity }}=0.97 ; \quad I^{2}=35 \%\right)$, without significant heterogeneity among the studies (Figure 2(b)); and overall mortality (22.8\% versus $19.4 \%$, OR 1.17 (95\% CI 0.79 to $\left.1.73) ; \quad p_{\text {heterogeneity }}=0.43 ; \quad I^{2}=34 \%\right)$, without significant heterogeneity among the studies (Figure 2(c)), respectively. Interestingly, the incidence of stroke was significantly lower in the TAVR group (3.8\%) compared to the SAVR group (7.9\%, OR 0.52 (95\% CI 0.27 to 0.98$) ; p_{\text {heterogeneity }}=0.04$; $I^{2}=0 \%$ ), without any significant heterogeneity among the evaluated studies (Figure 3(a)). Moreover, both groups did not differ in regard to acute kidney injury (9.7\% versus $8.7 \%$, OR 1.00 (95\% CI 0.49 to 2.07 ); $p_{\text {heterogeneity }}=0.99$; $I^{2}=46 \%$ ) (Figure $3(\mathrm{~b})$ ) or major adverse cardiovascular events (8.7\% versus $12.3 \%$, OR 0.60 , (95\% CI 0.28 to 1.32$)$; $p_{\text {heterogeneity }}=0.21 ; I^{2}=62 \%$ ) (Figure $3(\mathrm{c})$ ).

All secondary endpoints showed significant differences between both groups: the incidence of major bleeding was significantly lower in the TAVR group $(8.3 \%)$ compared to the SAVR group (15.3\%, OR 0.43 (95\% CI 0.19 to 0.97 ); 


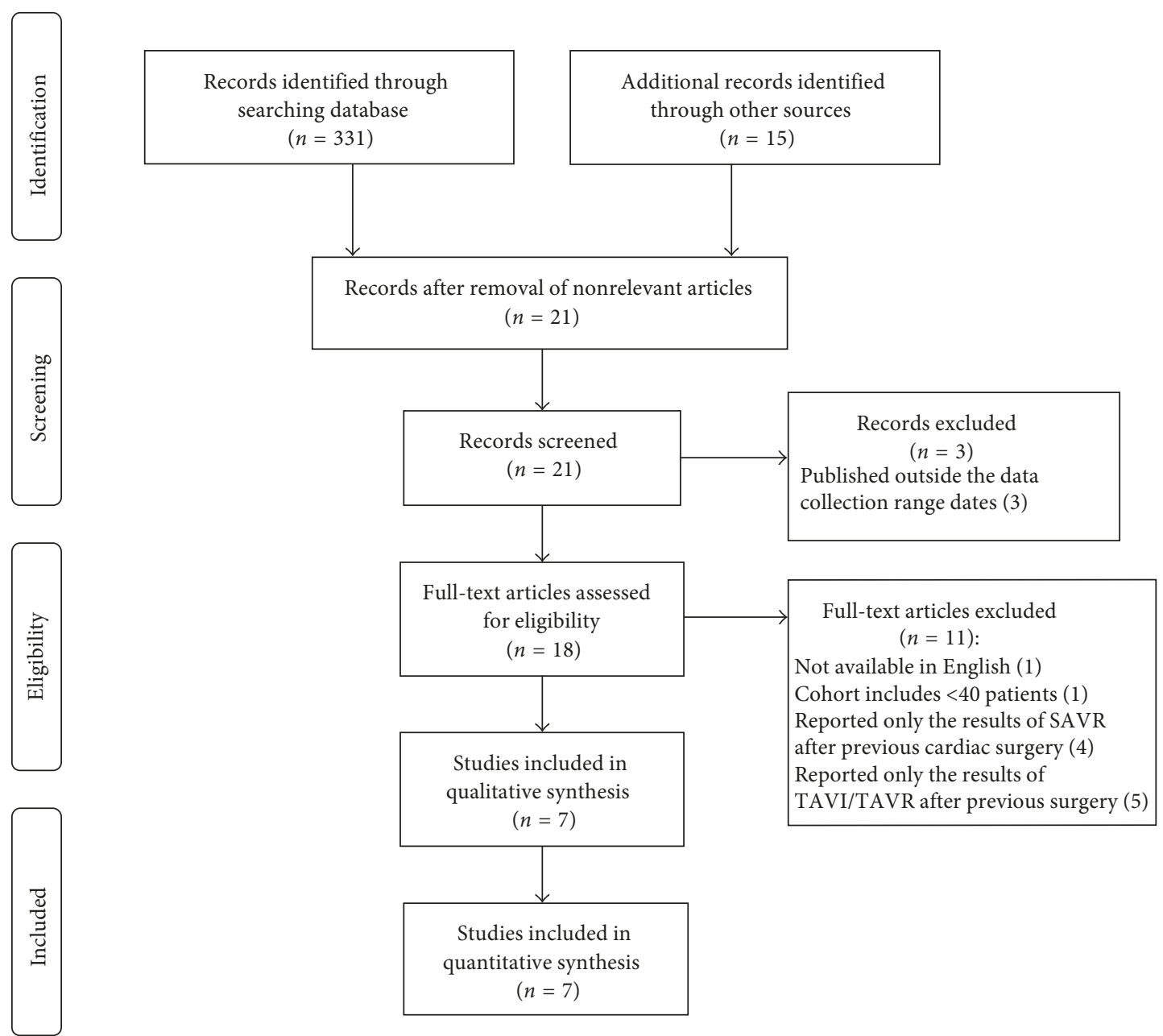

FIgURE 1: Study flowchart.

TABLE 1: Summery of the total incidences of the endpoints of the meta-analysis.

\begin{tabular}{|c|c|c|c|c|}
\hline & TAVR & SAVR & OR $(95 \% \mathrm{CI})$ & $p$ value \\
\hline \multicolumn{5}{|l|}{ Primary endpoints } \\
\hline Early mortality, $n(\%)$ & $29 / 565(5.1)$ & $32 / 583(5.5)$ & $0.89(0.49,1.62)$ & $p=0.7$ \\
\hline One-year mortality, $n(\%)$ & $50 / 432(11.6)$ & $53 / 450(11.8)$ & $1.01(0.59,1.72)$ & $p=0.97$ \\
\hline Overall mortality, $n(\%)$ & $129 / 565(22.8)$ & $113 / 583(19.4)$ & $1.17(0.79,1.73)$ & $p=0.43$ \\
\hline Stroke, $n(\%)$ & $16 / 417(3.8)$ & $35 / 443(7.9)$ & $0.52(0.27,0.98)$ & $p=0.04$ \\
\hline Acute kidney injury, $n(\%)$ & $55 / 565(9.7)$ & $38 / 435(8.7)$ & $1.00(0.49,2.07)$ & $p=0.99$ \\
\hline Major adverse events, $n(\%)$ & $39 / 450(8.7)$ & $59 / 479(12.3)$ & $0.60(0.28,1.32)$ & $p=0.21$ \\
\hline \multicolumn{5}{|l|}{ Secondary endpoints } \\
\hline Major bleeding, $n(\%)$ & $47 / 565(8.3)$ & $89 / 583(15.3)$ & $0.43(0.19,0.79)$ & $p=0.04$ \\
\hline Permanent pacemaker, $n(\%)$ & $64 / 565(11.3)$ & $23 / 583(3.9)$ & $2.79(1.24,6.28)$ & $p=0.01$ \\
\hline Moderate paravalvular leakage, $\mathrm{n}(\%)$ & $57 / 396(14.4)$ & $0 / 384(0)$ & $29.57(7.09,123.4)$ & $p<0.0001$ \\
\hline Severe paravalvular leakage, $n(\%)$ & $43 / 396(10.9)$ & $0 / 384(0)$ & $23.44(5.60,98.17)$ & $p<0.0001$ \\
\hline Procedural time (range), mean \pm SD & $(48 \pm 11)$ to $(225 \pm 60)$ & $(145 \pm 33)$ to $(384 \pm 92)$ & $-2.80(-3.65,-1.95)$ & $p<0.0001$ \\
\hline Hospital stay (range), mean \pm SD & $(5 \pm 0)$ to $(12 \pm 6)$ & $(8 \pm 0)$ to $(15 \pm 14)$ & $-0.38(-0.58,-0.19)$ & $p<0.0001$ \\
\hline
\end{tabular}

$p_{\text {heterogeneity }}=0.04 ; I^{2}=64 \%$ ); however, there was a significant heterogeneity among the evaluated studies (Figure 4(a)). Conversely, the incidence of permanent pacemaker implantation was significantly higher in the TAVR group (11.3\% versus 3.9\% patients, OR 2.79 (95\% CI 1.24 to 6.28 ); $p_{\text {heterogeneity }}=0.01 ; I^{2}=47 \%$ ) (Figure $4(\mathrm{~b})$ ). Moreover, the incidence of both mild-to-moderate and moderate-to-severe paravalvular leakage was significantly higher in the TAVR group (Figures 5(a) and 4(b)) with no PVL reported in the SAVR group. Procedural time (OR 2.80 (95\% CI 3.65 to 1.95); $p_{\text {heterogeneity }}<0.0001 ; I^{2}=90 \%$ ) (Figure $6(\mathrm{a})$ ) and hospital stay (OR 0.38 (95\% CI 0.58 to 0.19 ); $p_{\text {heterogeneity }}<0.0001$; $I^{2}=0 \%$ ) (Figure 6(b)) were significantly lower in the TAVR group. 


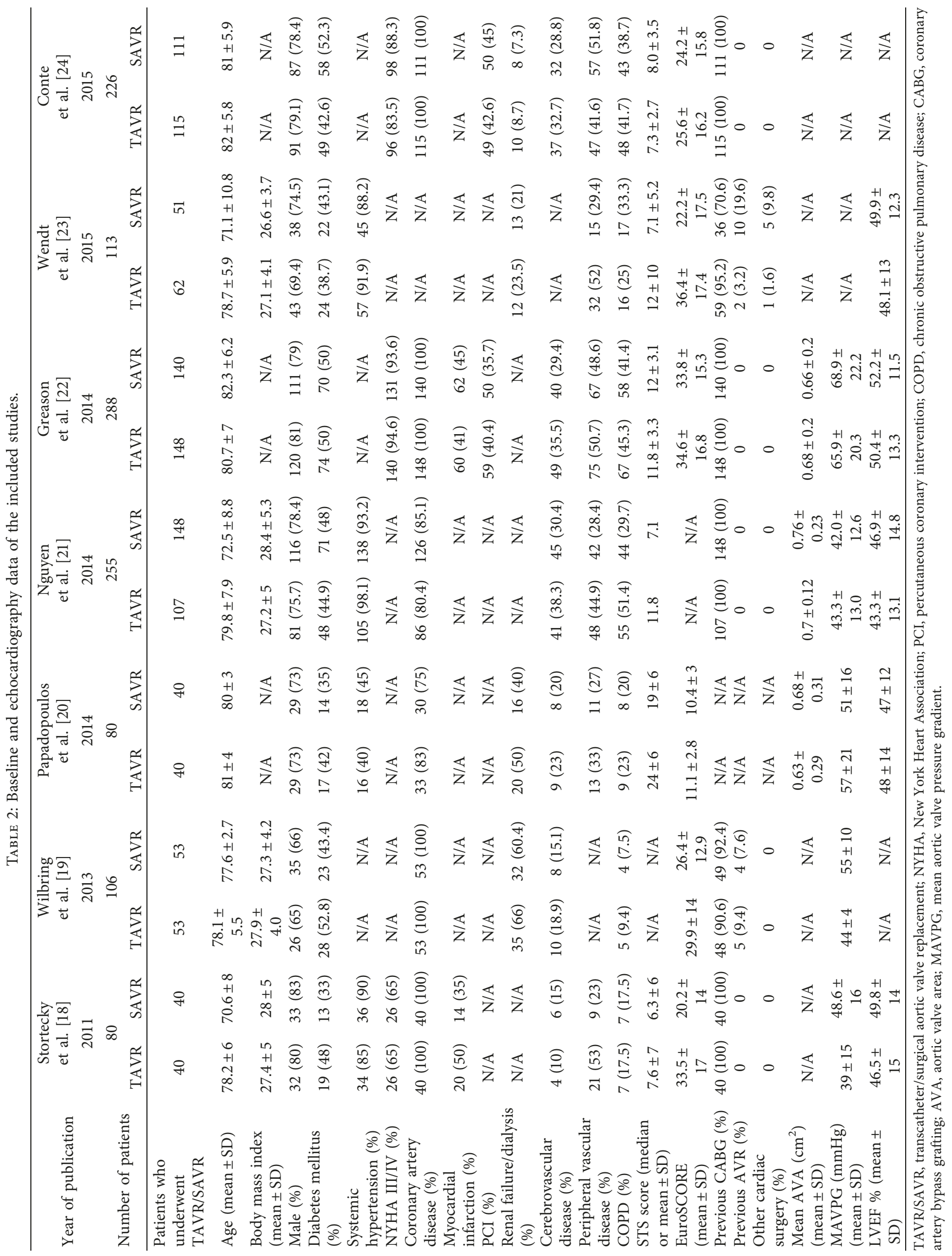




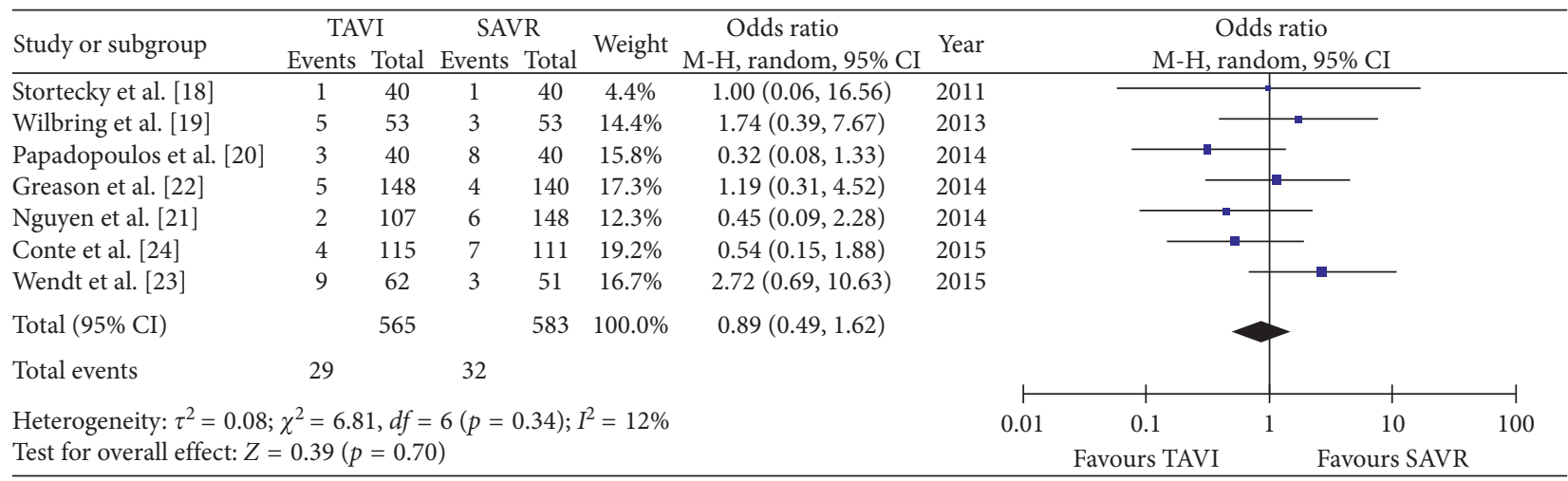

(a)

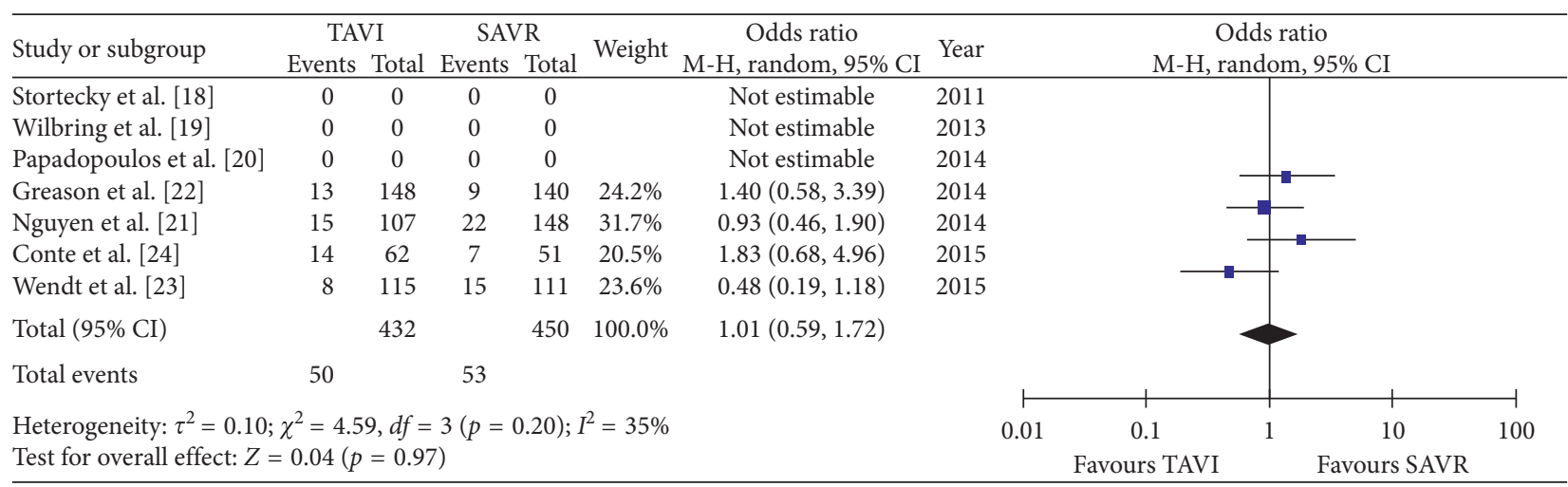

(b)

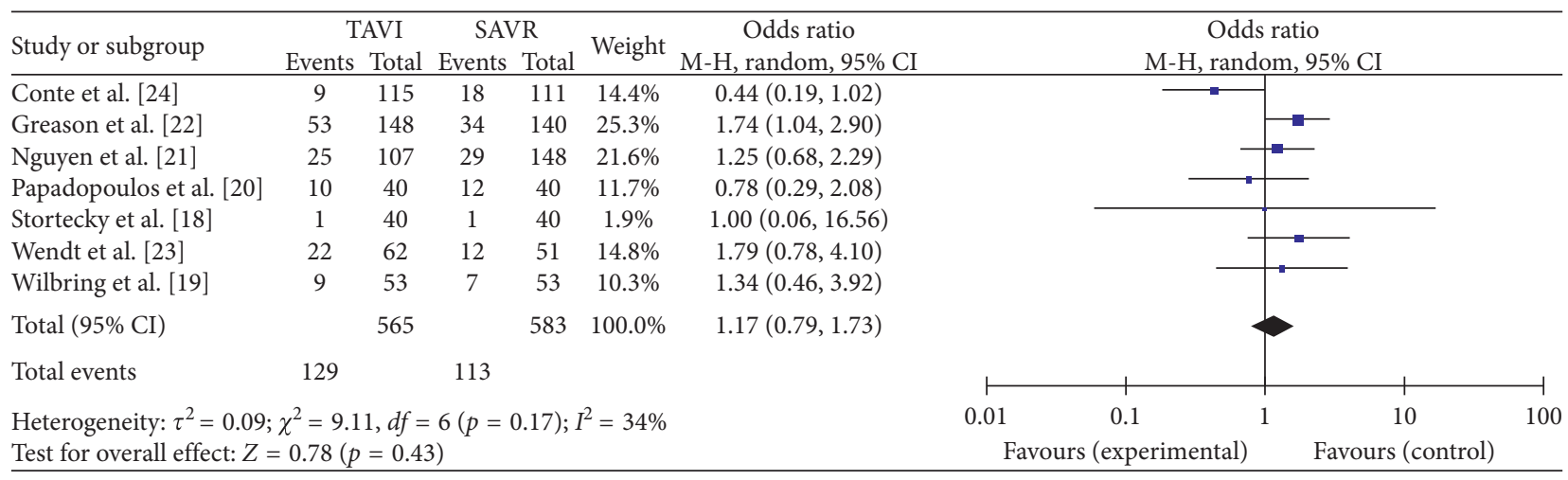

(c)

FIGURE 2: Meta-analytic comparison showing (a) early mortality rate between the TAVR and SAVR group, (b) one-year mortality between the TAVR and SAVR group, and (c) overall mortality between the TAVR and SAVR group.

\section{Discussion}

The current meta-analysis evaluates for the first time the outcomes of patients undergoing TAVR versus SAVR after a previous cardiac surgery. The main findings of this study were as follows: (1) there were no significant differences in early, oneyear, or overall mortality between both groups. (2) Interestingly, SAVR patients were more likely to experience postoperative stroke compared to TAVR patients. (3) There was no difference in postoperative acute kidney injury between both groups. (4) TAVR patients experienced significantly higher rates of pacemaker implantation and paravalvular leakage.
Before the TAVR era, surgical aortic valve replacement as a redo procedure in patients with previous CABG has been considered as the gold standard therapy for patients presenting with symptomatic aortic stenosis. The procedure, however, could be challenging due to patent bypass grafts. Mortality has been reported up to $20 \%$ in high-risk patients $[27,28]$. Although even lower mortality rates have been described [29], redo surgery is sometimes technically challenging due to severe adhesions with the risk of injury of the right ventricle, or patent graft injury, or the difficulty to achieve optimal myocardial protection even with the use of retrograde cardioplegia. Transcatheter aortic valve replacement has been 


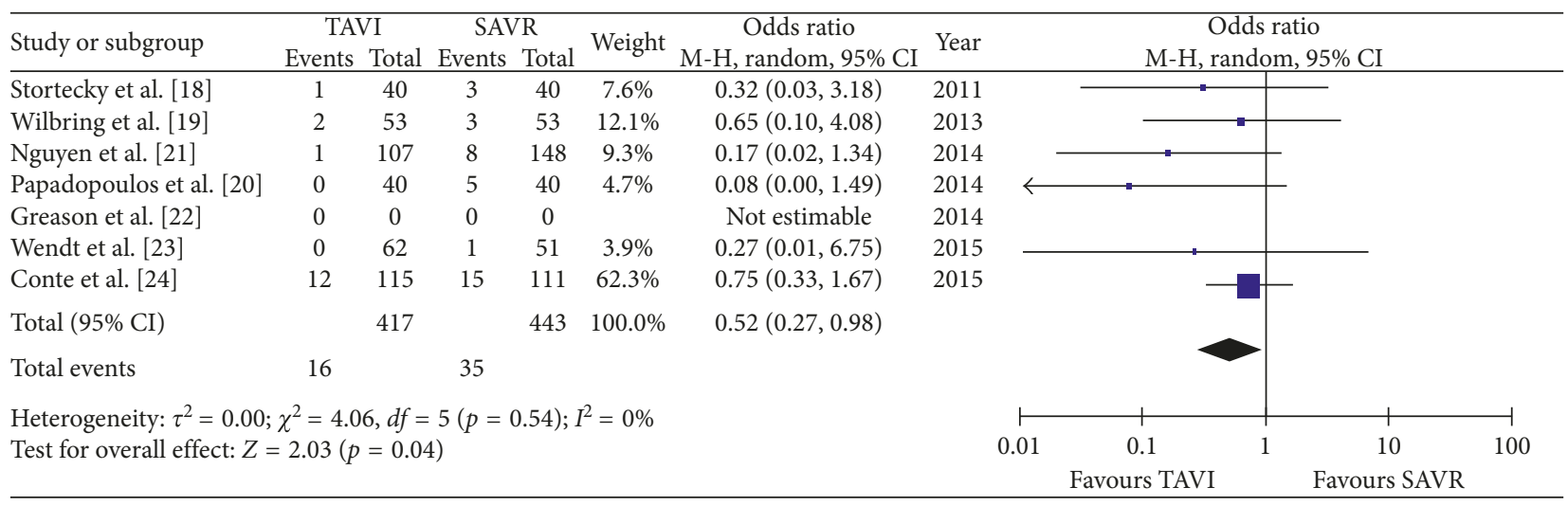

(a)

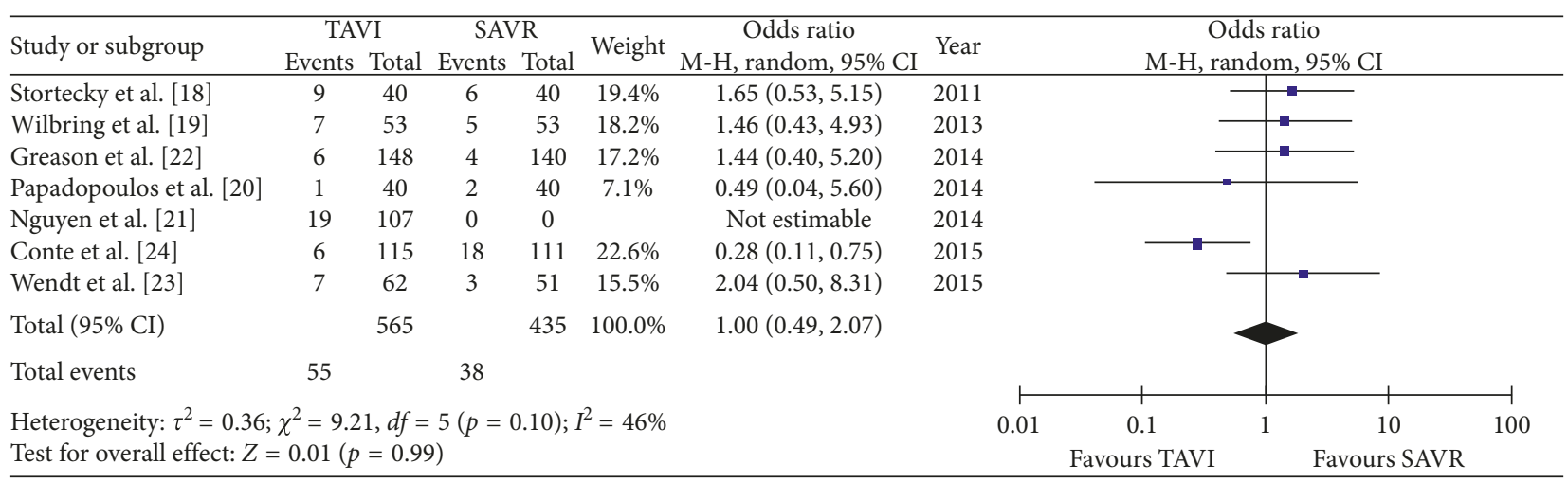

(b)

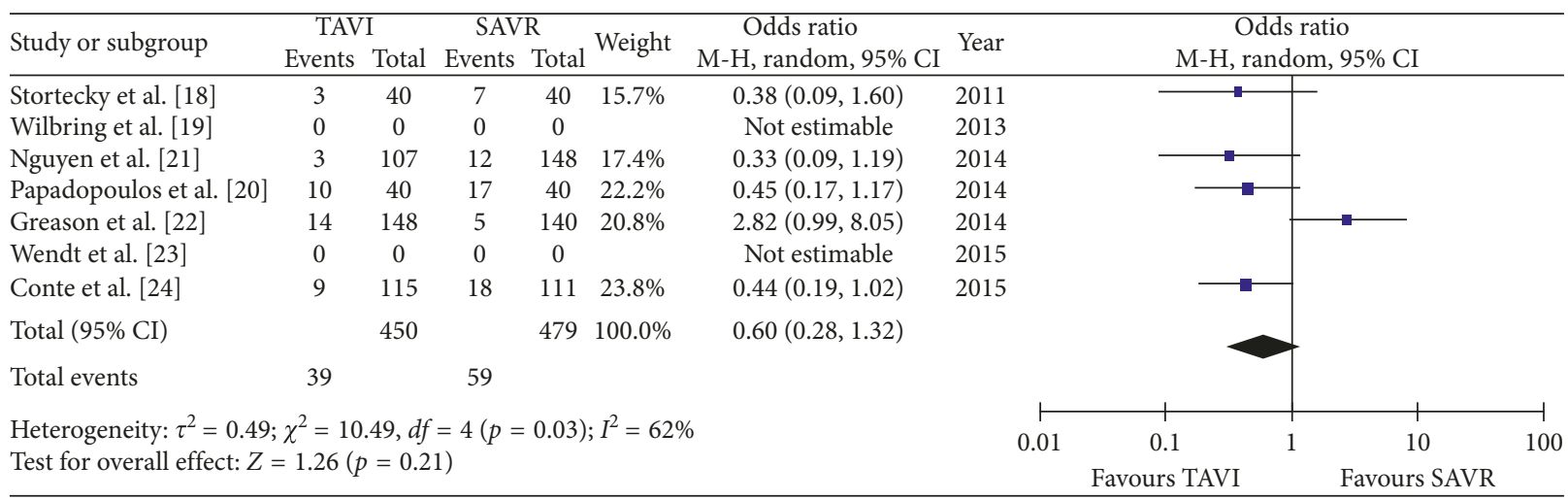

(c)

FIgURE 3: Meta-analytic comparison showing (a) incidence of stroke between the TAVR and SAVR group, (b) incidence of acute kidney injury between the TAVR and SAVR group, and (c) incidence of major adverse cardiovascular events between the TAVR and SAVR group.

established as an alternative therapy in patients with severe aortic stenosis, who were deemed to be at prohibitive risk for open-heart surgery. Moreover, TAVR presented promising results with lower intraprocedural complications and promising follow-up results in high-risk or even intermediate-risk patients [30].

Over the last years, there has been an ongoing debate about the advantages and disadvantages of TAVR over SAVR in primary aortic stenosis. The present study, however, aimed at evaluating the outcomes in a selected group of patients with previous cardiac surgery. Patients with prior cardiac surgery, by nature, show a higher risk, which is mainly reflected by the preoperative calculated risk scores. Interestingly, the current meta-analysis demonstrated a higher stroke rate in patients undergoing SAVR after prior cardiac surgery. Comparing those findings with previous reports, stroke rates vary between 5.7\% [6] and 8\% in the RECORD multicenter study [7]. Stroke might be caused by aortic cross clamping or calcium removal during surgical AVR, whereas in TAVR, the calcified aortic valve is pressed into the aortic wall, which could also cause stroke by calcified debris. Within the present metaanalysis, we did not observe any differences in the incidence of 


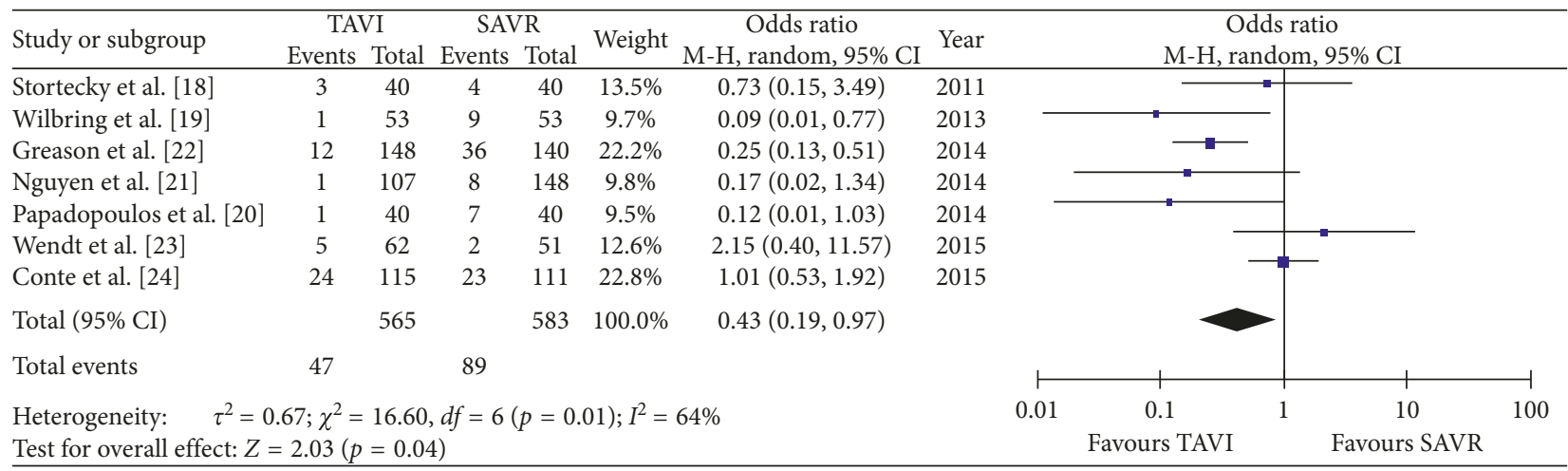

(a)

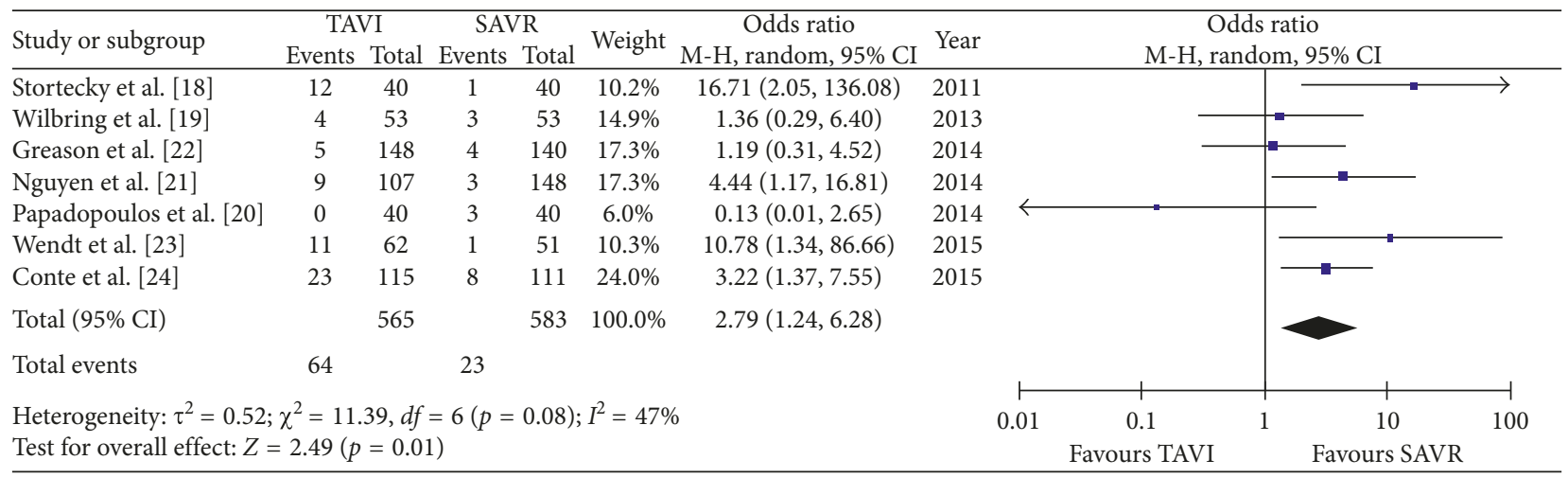

(b)

FIGURE 4: Meta-analytic comparison showing (a) incidence of major bleeding between the TAVR and SAVR group and (b) incidence of pacemaker implantation between the TAVR and SAVR group.

MACEs in both groups. There was no significant difference in the occurrence of acute kidney injury (AKI) rates in both groups, despite the use of contrast media in TAVR patients. In regard to acute kidney injury, previously published data demonstrated that, preoperative creatinine, the presence of peripheral vascular diseases, and blood transfusion are predictors for AKI after TAVR $[31,32]$. The contrast media was, however, not a predictor for AKI.

In regard to the secondary outcomes of the present metaanalysis, the redo SAVR group experienced more major bleeding (15.3\%) compared to TAVR patients (8.3\%). Those results are in accordance with the previously reported one that evaluated the risk of reexploration for bleeding in case of redo surgery due to dissection leaving a row area and/or injury of the heart or grafts due to severe adhesions. This increased risk of major bleeding events in the redo situation has been shown to be a predictor of 30-day mortality in the multivariable analysis by Vohra et al. [6]. Patients undergoing TAVR experienced more mild-to-moderate and moderate-to-severe PVL compared to SAVR. This has been also shown in the PARTNER trial, which additionally demonstrated that moderate-to-severe PVL was associated with higher 1-year mortality (cardiac and noncardiac) and rehospitalization after TAVR [33]. Even in patients with mild PVL, the mortality rate was higher than in those with no PVL [33] with a clear advantage for SAVR. Having said that, TAVR with thirdgeneration devices promises less PVL [34].
4.1. TAVR versus SAVR as a Redo Procedure. Currently, with improving devices, techniques, and encouraging recent results from TAVR in intermediate-risk patients $[4,5]$, the worldwide adoption of TAVR is becoming an important tool in the treatment of severe AS. However, patients with patent grafts presenting only with intermediate-risk scores are by nature a "higher risk" group due to possible harming of those patent grafts during redo surgery. Of note, patients undergoing TAVR or SAVR after previous CABG exhibited different mortality rates as calculated in the preoperative STS PROM or EuroSCORE. Moreover, previous reports discussed this important point and debate the role of STS or EuroSCORE in the decision-making between SAVR and TAVR. A previous study from Khaladj et al. evaluated the results of 349 patients who underwent SAVR after a history of CABG [16]. They reported that the early (inhospital or 30 -day) mortality was not higher than $5 \%$ compared to the calculated STS and logistic EuroSCORE of $10 \pm 4 \%$ and $32 \pm 21 \%$, respectively. Therefore, although all current riskscoring systems have been updated recently, both the STS PROM and EuroSCORE overestimated the risk of mortality in those patients $[35,36]$. The authors concluded that SAVR as a redo procedure after CABG can be performed with a lower mortality rate as predicted by STS or EuroSCORE [16].

In addition, those previous results were consistent with the results of the RECORD multicenter registry [7]. The 


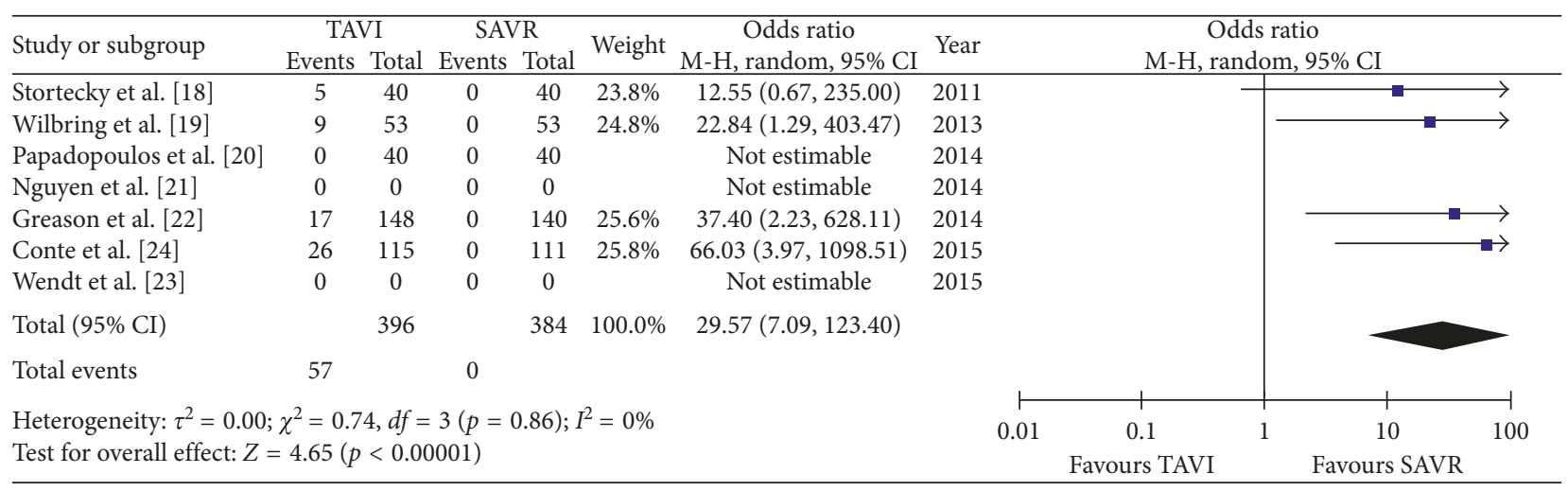

(a)

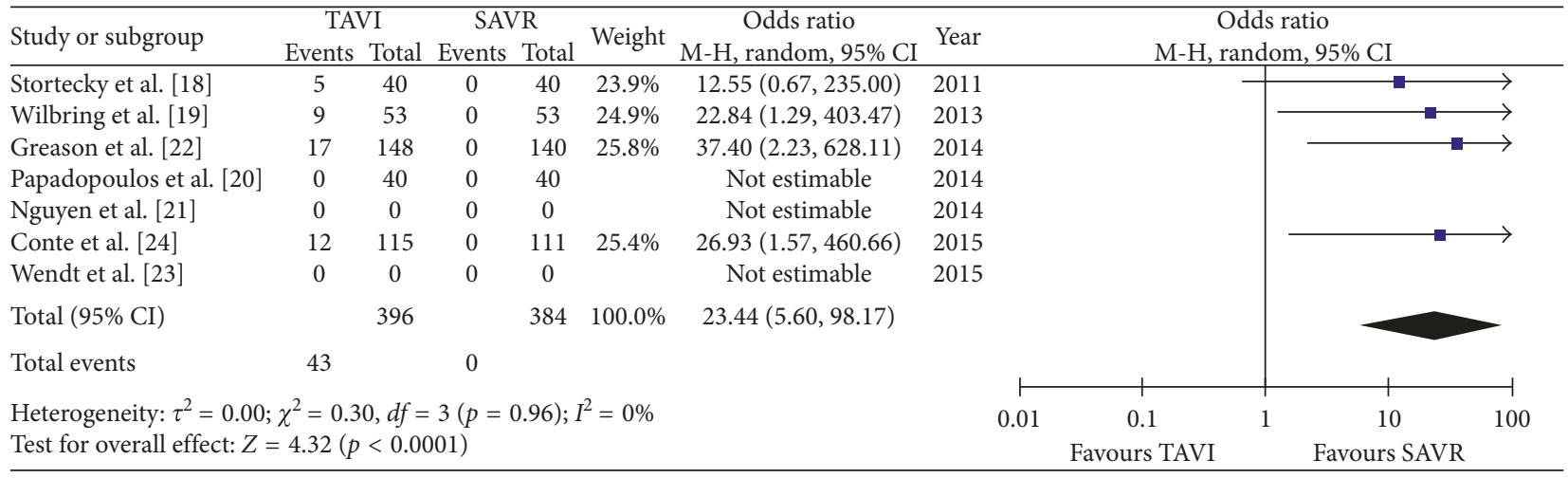

(b)

FIGURE 5: Meta-analytic comparison showing (a) incidence of mild-to-moderate paravalvular leakage between the TAVR and SAVR group and (b) incidence of moderate-to-severe paravalvular leakage between the TAVR and SAVR group.

investigators observed a lower early mortality of $4.4 \%$ in 113 patients who underwent an isolated SAVR after a history of CABG [7]. The authors concluded that a history of CABG should not be an indication for TAVR [7], although patients with prior CABG and especially those with patent grafts have an increased risk of graft injury. Interestingly, the present meta-analysis reported that TAVR patients experienced fewer strokes than SAVR patients in redo procedures. The decision, however, to choose either the TAVR or SAVR procedure in patients with prior surgery should be discussed in a "heart-team" and should include several factors including demographics, anatomical challenges, the presence of porcelain aorta, the number of patent grafts and, most importantly, the physical condition of the patient and, moreover, the individual patients' wish.

4.2. In Summary. The present meta-analysis showed no significant differences in early, one-year mortality, and overall mortality between TAVR and SAVR patients presenting with prior CABG surgery. SAVR patients demonstrated a lower rate of pacemaker and less mild-to-moderate PVL in comparison to TAVR patients in the redo situation. However, there was a higher rate of postprocedural stroke and bleeding in patients who underwent SAVR. TAVR offers an attractive, fast, and as safe alternative as SAVR for patients presenting with aortic stenosis after previous cardiac surgery, but the history of CABG per se should not be the only leading factor to decide for TAVR.

4.3. Study Limitations. The baseline characteristics were not similar in all included studies, and access site used for TAVR (e.g., transfemoral, transapical, transaortic, or transsubclavian access) was not mentioned in all studies. The evaluated endpoints depend mainly on the presence or absence of each event in the included studies; for example, early mortality is evaluated as inhospital mortality in some studies and as 30-day mortality in other studies; moreover, overall mortality was mentioned in the studies at different follow-up times which varies between 6 and 48 months, that is why it should not be considered as an accurate result in this meta-analysis. The type of cardioplegia used in SAVR was also not mentioned in all the included studies. In addition, all evaluated articles did not present the rate of potential graft injury during redo surgery and if a patent LIMA graft was clamped during redo surgery for aortic stenosis. Finally, the included studies did not present the cause and site of bleeding (e.g., graft injury or right ventricular injury). 


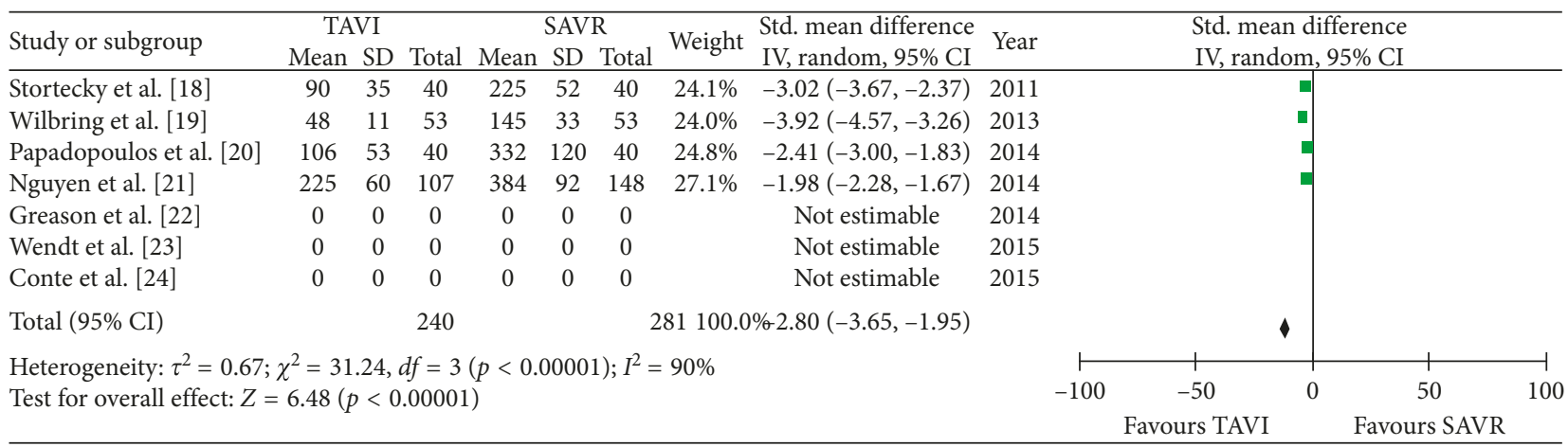

(a)

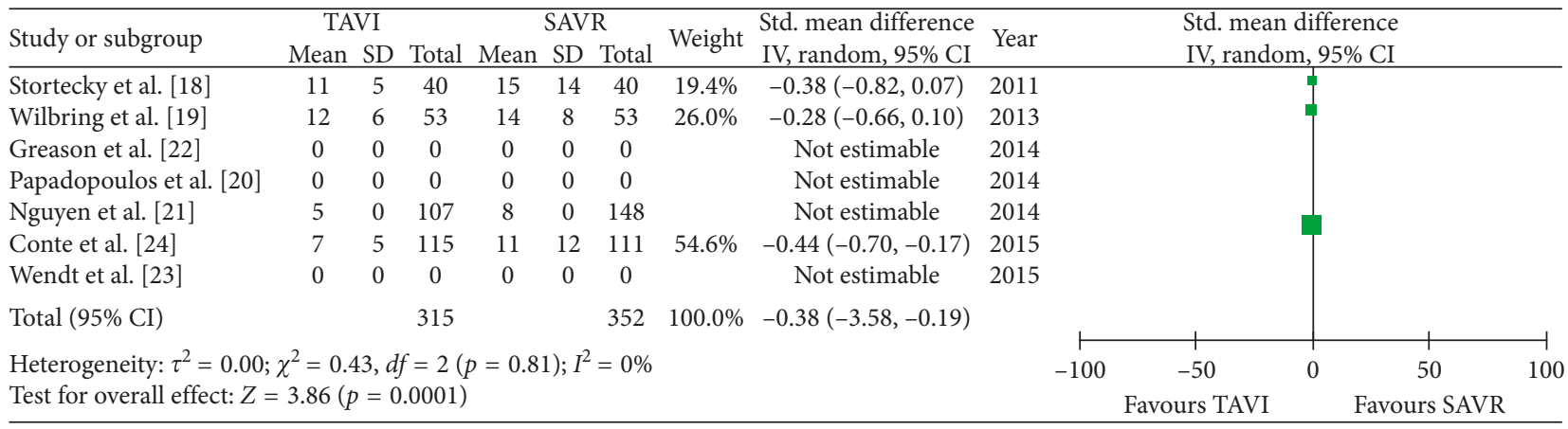

(b)

FIgURE 6: Meta-analytic comparison showing (a) procedural times in the TAVR and SAVR group and (b) length of hospital stays in the TAVR and SAVR group.

\section{Abbreviations}

AKI:

AS:

AVR:

CABG:

CI: Confidence interval

Acute kidney injury

Aortic stenosis

Aortic valve replacement

EuroSCORE: European System for Cardiac Operative Risk Evaluation

LVEF: Left ventricular ejection fraction

MACE: Major adverse cardiovascular events

OR: Odds ratio

PVL: $\quad$ Paravalvular leakage

TAVR: Transcatheter aortic valve replacement

SAVR: $\quad$ Surgical aortic valve replacement

STS PROM: Society of Thoracic Surgery

VARC: Valve Academic Research Consortium.

\section{Conflicts of Interest}

The authors declare that they have no conflicts of interest.

\section{Authors' Contributions}

Sharaf-Eldin Shehada and Yacine Elhmidi contributed equally and shared first authorship.

\section{Acknowledgments}

The authors are sincerely grateful to Miriam Radu, Andreas Sander, and Wolfgang Ristau (Institue of Quality Controlling, West-German Heart and Vascular Center Essen, University of Duisburg-Essen, Germany) for their generous effort and support in the data review to finish this work.

\section{References}

[1] American College of Cardiology, R. O. Bonow, B. A. Carabello et al., "ACC/AHA 2006 guidelines for the management of patients with valvular heart disease: a report of the American College of Cardiology/American Heart Association Task Force on Practice Guidelines (writing committee to revise the 1998 guidelines for the management of patients with valvular heart disease) developed in collaboration with the Society of Cardiovascular Anesthesiologists endorsed by the Society for Cardiovascular Angiography and Interventions and the Society of Thoracic Surgeons," Journal of the American College of Cardiology, vol. 48, no. 3, pp. el-e148, 2006.

[2] S. E. Shehada, Ö. Öztürk, M. Wottke, and R. Lange, "Propensity score analysis of outcomes following minimal access versus conventional aortic valve replacement," European Journal of Cardio-Thoracic Surgery, vol. 49, no. 2, pp. 464-470, 2015.

[3] S. E. Shehada, Y. Elhmidi, F. Mourad et al., "Minimal access versus conventional aortic valve replacement: a meta-analysis 
of propensity-matched studies," Interactive Cardiovascular and Thoracic Surgery, vol. 25, no. 4, pp. 624-632, 2017.

[4] V. H. Thourani, S. Kodali, R. R. Makkar et al., "Transcatheter aortic valve replacement versus surgical valve replacement in intermediate-risk patients: a propensity score analysis," The Lancet, vol. 387, no. 10034, pp. 2218-2225, 2016.

[5] M. J. Reardon, N. M. Van Mieghem, J. J. Popma et al., "Surgical or transcatheter aortic-valve replacement in intermediate-risk patients," New England Journal of Medicine, vol. 376, no. 14, pp. 1321-1331, 2017.

[6] H. A. Vohra, D. Pousios, R. N. Whistance et al., "Aortic valve replacement in patients with previous coronary artery bypass grafting: 10-year experience," European Journal of CardioThoracic Surgery, vol. 41, no. 3, pp. e1-e6, 2012.

[7] F. Biancari, F. Onorati, G. Mariscalco et al., "First-time, isolated surgical aortic valve replacement after prior coronary artery bypass surgery: results from the RECORD multicenter registry," Journal of Cardiac Surgery, vol. 29, no. 4, pp. 450-454, 2014.

[8] G. Ducrocq, N. Al-Attar, D. Himbert et al., "Early and midterm outcomes in patients undergoing transcatheter aortic valve implantation after previous coronary artery bypass grafting," European Journal of Cardio-Thoracic Surgery, vol. 41, no. 3, pp. 499-504, 2012.

[9] N. Papadopoulos, P. Ilioska, S. Fichtlscherer et al., "Transapical aortic valve implantation in patients with previous cardiac surgery," Annals of Thoracic Surgery, vol. 97, no. 1, pp. 37-42, 2014.

[10] S. Minha, M. A. Magalhaes, I. M. Barbash et al., "Impact of previous coronary artery bypass grafting on patients undergoing transcatheter aortic valve implantation for aortic stenosis," American Journal of Cardiology, vol. 113, no. 7, pp. 1222-1227, 2014.

[11] P. Castellant, R. Didier, E. Bezon et al., "Comparison of outcome of transcatheter aortic valve implantation with versus without previous coronary artery bypass grafting (from the FRANCE 2 Registry)," American Journal of Cardiology, vol. 116, no. 3, pp. 420-425, 2015.

[12] E. Leshem-Rubinow, Y. Abramowitz, A. Steinvil et al., "Outcomes of transfemoral transcatheter aortic valve implantation in patients with previous coronary bypass," American Journal of Cardiology, vol. 116, no. 3, pp. 431-435, 2015.

[13] S. E. Shehada, Y. Elhmidi, N. Puluca et al., "Impact of previous cardiac surgery in patients undergoing transcatheter aortic valve implantation: a systematic review," Journal of Cardiovascular Surgery, vol. 58, no. 5, pp. 787-793, 2017.

[14] T. A. Timek, Z. Turfe, R. L. Hooker et al., "Aortic valve replacement in octogenarians with prior cardiac surgery," Annals of Thoracic Surgery, vol. 99, no. 2, pp. 518-523, 2015.

[15] C. W. Akins, A. D. Hilgenberg, G. J. Vlahakes, J. C. Madsen, and T. E. MacGillivray, "Aortic valve replacement in patients with previous cardiac surgery," Journal of Cardiac Surgery, vol. 19, no. 4, pp. 308-312, 2004.

[16] N. Khaladj, M. Shrestha, S. Peterss et al., "Isolated surgical aortic valve replacement after previous coronary artery bypass grafting with patent grafts: is this old-fashioned technique obsolete?," European Journal of Cardio-Thoracic Surgery, vol. 35, no. 2, pp. 260-264, discussion 264, 2009.

[17] C. M. Vassileva, S. Aranki, J. Matthew Brennan et al., "Evaluation of the society of thoracic surgeons online risk calculator for assessment of risk in patients presenting for aortic valve replacement after prior coronary artery bypass graft: an analysis using the STS adult cardiac surgery database," Annals of Thoracic Surgery, vol. 100, no. 6, pp. 2109-2116, 2015.

[18] S. Stortecky, H. Brinks, P. Wenaweser et al., "Transcatheter aortic valve implantation or surgical aortic valve replacement as redo procedure after prior coronary artery bypass grafting," Annals of Thoracic Surgery, vol. 92, no. 4, pp. 1324-1330, discussion 1230-1231, 2011.

[19] M. Wilbring, S.-M. Tugtekin, K. Alexiou, G. Simonis, K. Matschke, and U. Kappert, "Transapical transcatheter aortic valve implantation vs conventional aortic valve replacement in high-risk patients with previous cardiac surgery: a propensity-score analysis," European Journal of CardioThoracic Surgery, vol. 44, no. 1, pp. 42-47, 2013.

[20] N. Papadopoulos, N. Schiller, S. Fichtlscherer et al., "Propensity matched analysis of longterm outcomes following transcatheter based aortic valve implantation versus classic aortic valve replacement in patients with previous cardiac surgery," Journal of Cardiothoracic Surgery, vol. 9, no. 1, p. 99, 2014.

[21] T. C. Nguyen, V. C. Babaliaros, S. A. Razavi et al., "Transcatheter aortic valve replacement has improved short-term but similar midterm outcomes in isolated aortic valve replacement after prior coronary artery bypass grafting," Annals of Thoracic Surgery, vol. 98, no. 4, pp. 1316-1324, 2014.

[22] K. L. Greason, V. Mathew, R. M. Suri et al., "Transcatheter versus surgical aortic valve replacement in patients with prior coronary artery bypass graft operation: a PARTNER trial subgroup analysis," Annals of Thoracic Surgery, vol. 98, no. 1, pp. 1-8, discussion 7-8, 2014.

[23] D. Wendt, F. Al-Rashid, P. Kahlert et al., "Conventional aortic valve replacement or transcatheter aortic valve implantation in patients with previous cardiac surgery," Journal of Cardiology, vol. 66, no. 4, pp. 292-297, 2015.

[24] J. V. Conte, T. G. Gleason, J. R. Resar et al., "Transcatheter or surgical aortic valve replacement in patients with prior coronary artery bypass grafting," Annals of Thoracic Surgery, vol. 101, no. 1, pp. 72-79, 2015.

[25] D. Moher, A. Liberati, J. Tetzlaff, and D. G. Altman, "Preferred reporting items for systematic reviews and meta-analyses: the PRISMA statement," Annals of Internal Medicine, vol. 151, no. 4, pp. 264-269, 2009.

[26] A. P. Kappetein, S. J. Head, P. Généreux et al., "Updated standardized endpoint definitions for transcatheter aortic valve implantation: the Valve Academic Research Consortium-2 consensus document," European Heart Journal, vol. 33, no. 19, pp. 2403-2418, 2012.

[27] S. F. Fighali, A. Avendano, M. A. Elayda et al., "Early and late mortality of patients undergoing aortic valve replacement after previous coronary artery bypass graft surgery," Circulation, vol. 92, no. 9, pp. II163-II168, 1995.

[28] J. A. Odell, C. J. Mullany, H. V. Schaff, T. A. Orszulak, R. C. Daly, and J. J. Morris, "Aortic valve replacement after previous coronary artery bypass grafting," Annals of Thoracic Surgery, vol. 62, no. 5, pp. 1424-1430, 1996.

[29] J. P. Verhoye, F. Merlicco, I. M. Sami et al., "Aortic valve replacement for aortic stenosis after previous coronary artery bypass grafting: could early reoperation be prevented?," Journal of Heart Valve Disease, vol. 15, no. 4, pp. 474-478, 2006.

[30] Y. Elhmidi, S. Bleiziffer, N. Piazza et al., "Long-term results after transcatheter aortic valve implantation: what do we know today?," Current Cardiology Reviews, vol. 9, no. 4, pp. 295-298, 2013. 
[31] Y. Elhmidi, S. Bleiziffer, N. Piazza et al., "Incidence and predictors of acute kidney injury in patients undergoing transcatheter aortic valve implantation," American Heart Journal, vol. 161, no. 4, pp. 735-739, 2011.

[32] Y. Elhmidi, S. Bleiziffer, M.-A. Deutsch et al., "Acute kidney injury after transcatheter aortic valve implantation: incidence, predictors and impact on mortality," Archives of Cardiovascular Diseases, vol. 107, no. 2, pp. 133-139, 2014.

[33] S. Kodali, P. Pibarot, P. S. Douglas et al., "Paravalvular regurgitation after transcatheter aortic valve replacement with the Edwards sapien valve in the PARTNER trial: characterizing patients and impact on outcomes," European Heart Journal, vol. 36, no. 7, pp. 449-456, 2015.

[34] D. Wendt, F. Al-Rashid, P. Kahlert et al., "low incidence of paravalvular leakage with the balloon-expandable sapien 3 transcatheter heart valve," Annals of Thoracic Surgery, vol. 100, no. 3, pp. 819-825, discussion 825-826, 2015.

[35] D. Wendt, M. Thielmann, P. Kahlert et al., "Comparison between different risk scoring algorithms on isolated conventional or transcatheter aortic valve replacement," Annals of Thoracic Surgery, vol. 97, no. 3, pp. 796-802, 2014.

[36] D. Wendt, B. R. Osswald, K. Kayser et al., "Society of Thoracic Surgeons score is superior to the EuroSCORE determining mortality in high risk patients undergoing isolated aortic valve replacement," Annals of Thoracic Surgery, vol. 88, no. 2, pp. 468-474, discussion 474-475, 2009. 


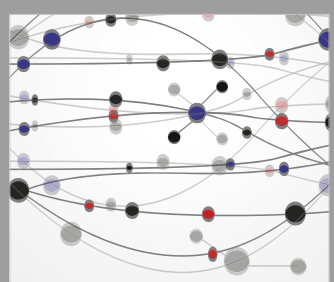

The Scientific World Journal
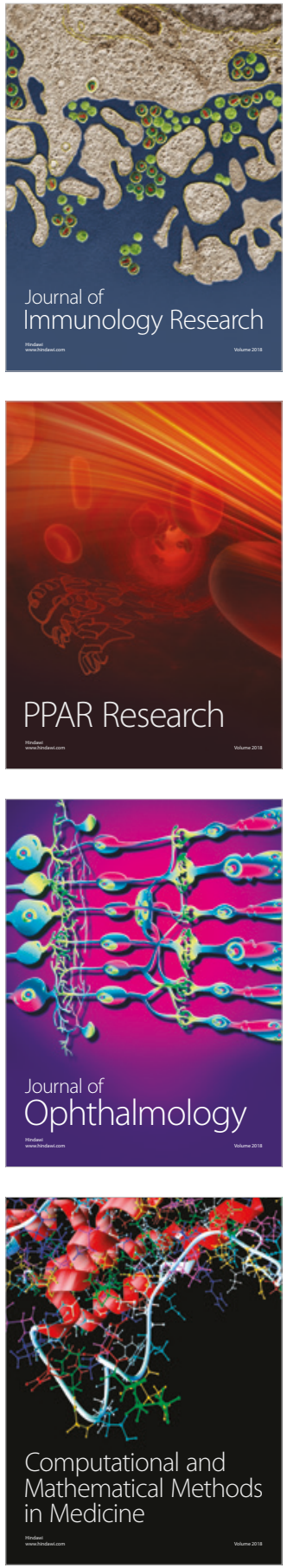

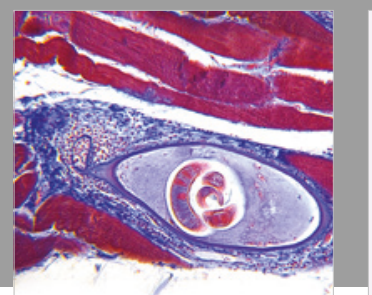

Gastroenterology Research and Practice

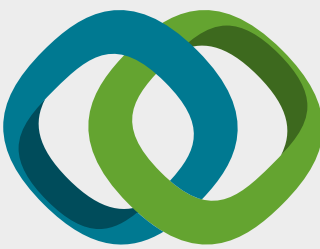

\section{Hindawi}

Submit your manuscripts at

www.hindawi.com
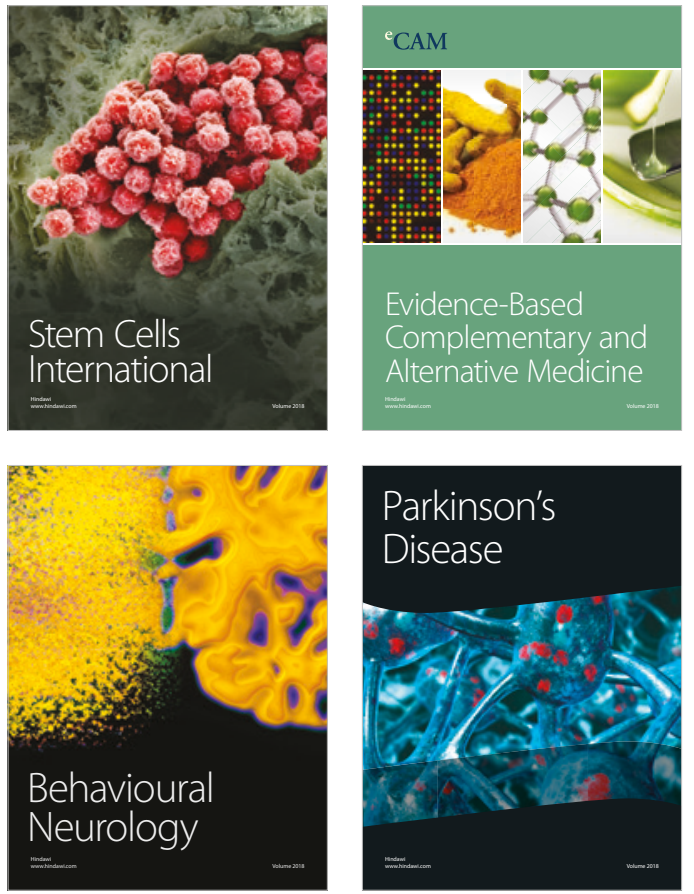

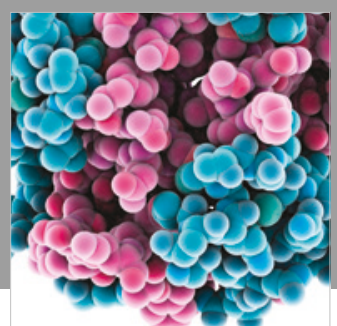

ournal of

Diabetes Research

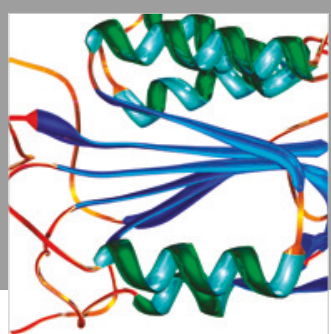

Disease Markers
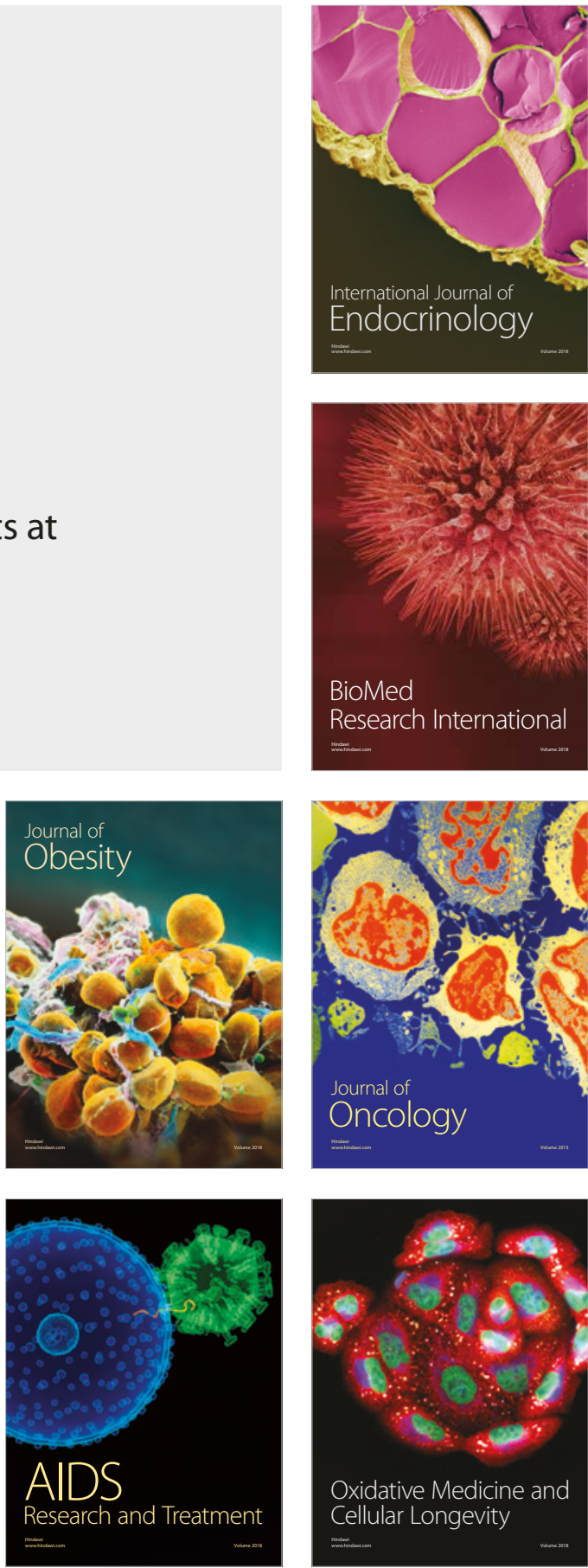